\title{
PERCUTANEOUS ULTRASOUND-GUIDED PUNCTURE AND CATHETER DRAINAGE METHODS IN THE TREATMENT OF FLUID COLLECTIONS FOLLOWING ACUTE PANCREATITIS
}

DOI: $10.36740 /$ WLek202108103

\author{
Tetiana Formanchuk', Hryhoriy Lapshyn², Oleg Voznyuk³ , Andrii Formanchuk' ${ }^{1}$, Andrii Zhmur ${ }^{1}$ \\ 'NATIONAL PIROGOV MEMORIAL MEDICAL UNIVERSITY, VINNYTSIA, UKRAINE \\ 2UNIVERSITY MEDICAL CENTER LUBECK, LUBECK, GERMANY \\ ${ }^{3}$ VINNYTSIA CITY EMERGENCY CLINICAL HOSPITAL, VINNYTSIA, UKRAINE
}

\begin{abstract}
The aim: Improve the treatment outcomes of patients with fluid collections following acute pancreatitis using an ultrasound-guided puncture and catheter drainage methods. Materials and methods: 67 patients with acute pancreatitis complicated by fluid collections were divided into two groups. The first group (comparison group) consisted of 32 patients who underwent percutaneous ultrasound-guided puncture and catheter drainage interventions in addition to conservative therapy. The second group (control group) consisted of 35 patients receiving conservative therapy. The age of patients was from 18 to 77 years. In the comparison group among 32 patients there were 19 women and 13 men, the average age consisted $48.2 \pm 2.2$ years. In the control group among 35 patients there were 21 women and 14 men, the average age of patients consisted $47.1 \pm 2.3$ years. Results: The mortality rate in the comparison group was $2(6.2 \%)$ cases, in the control group $-4(11.4 \%)$ cases ( $<<0.05)$. Infection of fluid collections developed in 2 (6.2\%) patients of the comparison group and in 5 (14.3\%) patients of the control group. The average length of stay in the hospital of patients in the comparison group was $24.13 \pm 2.17$ days, in the control group $28.11 \pm 1.05$ days $(p<0.05)$. Also in the comparison group there was a faster normalization of clinical and laboratory indicators (level of leukocytes, serum amylase, $($-reactive protein) $(p<0,05)$.

Conclusions: the use of percutaneous ultrasound-guided puncture and catheter drainage methods has reduced mortality and improved treatment outcomes in patients with acute pancreatitis complicated by fluid collections
\end{abstract}

KEY WORDS: acute pancreatitis, minimally invasive methods, drainage of fluid collection, open necrosectomy

Wiad Lek. 2021;74(8):1794-1799

\section{INTRODUCTION}

Fluid collections around the pancreas belong to complications of acute pancreatitis and usually follow its moderate and severe course. According to the updated classification by Atlanta 2012, the local complications of acute pancreatitis include four subcategories of fluid collections: acute peripancreatic fluid collection, pancreatic pseudocyst, acute necrotic collection and walled-off necrosis. In the updated classification much attention is paid to the differentiation of these concepts. The criteria for differences in these concepts are: the nature of the content of accumulations - a liquid component or fluid with necrotic detritus, the accumulation is localized in the gland or beyond it, the presence or absence of a formed capsule [1].

In recent decades, many guidelines have been developed for the management of acute necrotic pancreatitis, but for a long time no guidance has been offered on the use of minimally invasive necrosectomy techniques. In 2012, the medical community was presented with the results of a multidisciplinary consensus conference on interventions in acute necrotic pancreatitis, which was attended by international experts in many disciplines. According to the results of the conference, the primary indications for interventions are infected necrotic pancreatitis, much less often - symptomatic correction in sterile necrotizing pancreatitis. The recommendations of the conference are to postpone interventions as long as possible, not earlier than 4 weeks after the onset of the disease. Gradual application of percutaneous drainage in combination with minimally invasive video-assisted retroperitoneal debridement and endoscopic necrosectomy showed better results compared to open necrosectomy and are the methods of choice [2].

In the available literature we found a lot of research that confirms the advantages of mini-invasive interventions before performing open necrosectomy in patients with necrotizing pancreatitis [3-6].

The use of minimally invasive interventions can delay surgery for acute pancreatitis. In all the sources analyzed by us over the past 5 years, it is reported that the delay of surgery in acute pancreatitis is accompanied by a significant reduction in mortality. It is necessary to try to postpone the operation to the so-called "cold period". Currently, the guidelines of the World Society of Emergency Surgery 2019 recommend postponing surgery for more than 4 weeks from the onset of the disease. This is the time required for a clear demarcation between viable and necrotic tissues 
of the pancreas, which minimizes trauma during surgery and reduces the risk of bleeding. This significantly reduces hospital mortality [7].

Ultrasound-guided puncture and catheter drainage methods and endoscopic methods are now recognized as first-line interventions in the treatment of fluid collections [8]. Evacuation of fluid containing pro-inflammatory cytokines reduces endotoxicosis and the development of multiple organ failure, promotes rapid normalization of inflammation.

Numerous studies have shown the high efficiency of minimally invasive interventions compared to conservative therapy. Xiang Li and colleagues (2020) based on a retrospective analysis of the results of treatment of 111 patients with acute pancreatitis with fluid accumulation showed a weakening of the inflammatory response (C-reactive protein, procalcitonin, leukocytes, amylase / lipase) and a lower level of multiple organ failure in the group with percutaneous fluid intake compared with the group with conservative therapy [9]. Krivoruchko et al. applied a differentiated approach using minimally invasive technologies, which reduced the number of postoperative complications from $38.1 \%$ to $15.2 \%$, and reduced mortality from $6.6 \%$ to $1.3 \%$ [10].

Fluid accumulations with a homogeneous fluid character, such as pancreatic pseudocyst and acute peripancreatic fluid accumulation, are easier to evacuate. At the same time, the presence of necrotic impurities in liquid accumulations requires a multidisciplinary approach and is often an intermediate stage in treatment $[8,11]$.

Percutaneous drainage of pancreatic and peripancreatic necrosis may be finished by the placement of a single or, if necessary, multiple catheters, the diameter of which may also vary, depending on the homogeneity of the contents and the purpose: evacuation of only the liquid part, debridement, irrigation. Also, percutaneous drainage can be performed as a primary intervention or can be performed in combination with other methods of the residual or infected fluid-necrotic accumulations removing. Usually, catheters from 12 to $30 \mathrm{Fr}$ are used, placing them transperitoneally or retroperitoneally. The last variant is a priority because it prevents abdominal contamination and other complications [2]. Fluid collections with a volume more than $500 \mathrm{ml}$ and purulent cavities are recommended to be drained percutaneously with the subsequent sanitation of the cavity with solutions of antiseptics up to 4 times a day during the day [10].

\section{THE AIM}

The aim of this study was to improve the treatment outcomes of patients with fluid collections following acute pancreatitis using an ultrasound-guided puncture and catheter drainage methods.

\section{MATERIALS AND METHODS}

The results of treatment of 67 patients with acute pancreatitis complicated by fluid collections, who were hospitalized in the surgical department of the Vinnytsya city clinical emergency hospital from January 2015 to June 2020. The diagnosis of acute pancreatitis was formulated according to the guidelines of the World Society of Emergency Surgery in 2019 as the presence of at least two of the following criteria: abdominal pain of appropriate localization and nature, biochemical signs of pancreatitis (three times increased and above the upper limit of normal serum amylase and / or lipase), the corresponding changes in the pancreas according to the results of imaging) [4]. The study was performed in accordance with all the rules of bioethics. Informed written consent to data processing was obtained from all patients. An updated Atlanta 2012 classification was used to classify patients according to the type of fluid collection. The choice of surgical tactics in the clinic was based on the guidelines of the World Society of Emergency Surgery 2019, which recommended postponing surgery for more than 4 weeks from the onset of the disease.

All patients with fluid collections following acute pancreatitis were divided into two groups depending on the method of treatment. The first group (comparison group) consisted of 32 patients who underwent percutaneous ultrasound-guided puncture and drainage interventions in addition with infusion therapy. The second group (control group) consisted of 35 patients receiving conservative therapy. The age of the patients was from 18 to 77 years. In the comparison group among 32 patients there were 19 women and 13 men, the average age of patients was $48.2 \pm$ 2.2 years. In the control group among 35 patients there were 21 women and 14 men, the mean age of patients was $47.1 \pm 2.3$ years

All patients with fluid collections following acute pancreatitis underwent a comprehensive clinical and laboratory examination, fibrogastroduodenoscopy, plan radiography of the abdominal cavity and chest, as a screening method of visualization of the pancreas - an ultrasound method was used, if needed - contrast-enhanced tomography of the abdominal cavity and retroperitoneal space was used. Ultrasound examination of the abdominal cavity and retroperitoneal space was performed on the device Siemens Healthineers, ACUSON Juniper, convex sensor with a frequency $5,5 \mathrm{MHz}$ and linear sensor with a frequency 6,5-13 MHz. Computer tomography of the abdominal cavity and retroperitoneal space was performed on a Siemens Somatom go.up with a tomograph step of $2 \mathrm{~mm}$, with intravenous administration ultravist $370 \mathrm{mg} / 100 \mathrm{ml}$.

Conservative therapy included gastrointestinal decompression, fasting for the first days followed enteral nutrition with balanced mixtures, infusion antispasmodic, antisecretory, detoxification, anticoagulant, antibacterial therapy, restoration of water balance, electrobalance correction. If adequate analgesia was required, patients underwent catheterization of the epidural space.

In the comparison group, the puncture of liquid accumulations was performed under the local anesthesia under ultrasound control, using a linear sensor with a frequency 7.5 MHz, operated in gray-scale mode. Indications for per- 
cutaneous puncture-drainage method were: the presence of a liquid collection with a volume $500 \mathrm{ml}$ or more or other liquid collection which increases intra-abdominal pressure (more than $15 \mathrm{~mm} \mathrm{Hg}$ ) and a rapid increase in fluid collection in the dynamics of ultrasonography (increase in fluid height more than $1.5 \mathrm{~cm} /$ day). For the puncture were used puncture needles 16-20 G (Balton, Poland). For drainage a sets for percutaneous drainage 6-20 G (firm "Balton", Poland) were used. The received fluid was sent for microscopic, bacteriological examination and determination of the level of diastase. Criteria for infection of fluid collections were positive results of bacteriological studies of fluid, as well as imaging data with the appropriate clinical and laboratory picture.

The puncture was performed in the operating room. Before that, an ultrasound examination was performed with a linear sensor, the location of the fluid accumulation and the place of its closest adjacent to the abdominal wall was determined. The presence of any part of the intestine, stomach, liver between the sensor and the fluid accumulation was considered as a contraindication for the puncture site. Under the local anesthesia, the tip of the puncture needle was inserted under the ultrasound guidance towards the fluid collection, using the "hands-free" technique, which allowed constant monitoring of the needle position during the procedure and prevent iatrogenic damage of adjacent structures.

The duration of pain, hospital stay, timing of GIT-motoric recovery, normalization of leukocytes, serum amylase / diastase, C-reactive protein, the features of echosemiotics at different times were compared in the groups. Statistical data processing was performed by the method of mathematical statistics with the software StatSoft Statistica 6.0 and Microsoft Office Excel.

\section{RESULTS}

Percutaneous ultrasound-guided puncture and catheter drainage methods were performed in 32 patients with fluid collections due to acute pancreatitis. In 24 (75.0\%) patients this method was performed within 4 weeks from the onset of the disease, in 8 (25.0\%) patients - after 4 weeks from the onset of the disease. In 11 (34.4\%) patients was sufficient to perform percutaneous puncture of the fluid collections, in 21 (65.6\%) patients a catheter drainage of the fluid collection was required after percutaneous puncture. Among the 21 patients who required catheter drainage, 13 patients underwent single drainage, 8 patients required paired drainage to re-draine residual fluid collections and for debridement. In 12 (37.5\%) patients, the percutaneous ultrasound-guided puncture and catheter drainage methods were performed as the first step before open necrosectomy, which allowed to postpone the performance of open necrosectomy later than 4 weeks from the onset of the disease.

The duration of pain in the comparison group was $8.09 \pm$ 0.73 days, in the control group $11.06 \pm 0.68$ days $(\mathrm{p}<0.001)$. The average length of stay in the hospital of patients in the comparison group was $24.13 \pm 2.17$ days, in the control group $-28.11 \pm 1.05(\mathrm{p}<0.05)$. Terms of recovery of gastrointestinal motility in the comparison group $12.5 \pm$ 2.7 days, in the control group $14.11 \pm 1.13$ days ( $p>0.05$ ).

Normalization of the leukocyte level in the comparison group was faster than in the control group - by $11.25 \pm$ 2.04 days and $13.2 \pm 1.47$ days appropriately $(\mathrm{p}<0.05)$. Normalization of serum amylase levels in the comparison group occurred on day $12.31 \pm 2.26$, in the control group on day $14.8 \pm 1.43$ appropriately $(\mathrm{p}<0.001)$. The level of $\mathrm{C}$-reactive protein in the comparison group was normalized on $12.56 \pm 2.04$ days, in the control group on $14.62 \pm$ 1.65 days $(\mathrm{p}>0.05)$.

Infection of fluid accumulations developed in 2 (6.2\%) patients of the comparison group and in $5(14.3 \%)$ patients of the control group $(\mathrm{p}<0.05)$. The mortality rate in the comparison group consisted $2(6.2 \%)$ cases, in the control group - $4(11.4 \%)$ cases.

\section{DISCUSSION}

At the initial stage, the formation of fluid collection was limited to infusion therapy and ultrasound monitoring in the dynamics. No punctures were performed, believing that accumulations up to $500 \mathrm{ml}$ did not require surgery and with a favorable course on the basis of adequate infusion therapy are well regressed.

In case of inefficiency of conservative therapy and increased fluid collection the appearance of complaints of a feeling of distension in the epigastrium, signs of gastro- and / or duodenostasis, vomiting, often shortness of breath occured. The re-sonogram of the abdominal cavity and retroperitoneal space showed an increase in the height of the strip of fluid, its displacement of neighboring organs, the spread of fluid along the interstitial spaces (Fig. 1).

The appearance of signs of gastro- and / or duodenostasis on the basis of a rapid increase of fluid accumulation, regardless of the presence of signs of infection and regardless of the time from the onset of the disease, was regarded as indications for ultrasound-guided puncture and possible subsequent catheter drainage of fluid.

During acute fluid collections puncturing, much attention was paid to the nature of the evacuated fluid. If fluid collection appeared in the early period after the onset of disease (up to 4 weeks), fluid was of the homogeneous consistency, transparent hemorrhagic nature, no additional inclusions, no viscosity, without connection with the pancreatic duct according to ultrasonography, volume of fluid collection was up to $500 \mathrm{ml}$ - the ultrasound-guided puncture of this fluid collection was performed. In $54.5 \%$ of cases (6 patients) it was enough to perform a single puncture of the fluid collection, in $45.5 \%$ of cases (5 patients) there was a need for repeated punctures of residual fluid collections. If the indications for puncture occurred on 3-5 weeks after the onset of the disease, this significantly increased the probability of infection, the evacuated fluid had a heterogeneous, viscous consistency, with inclusions of detritus, turbid, fluid collection according to ultraso- 


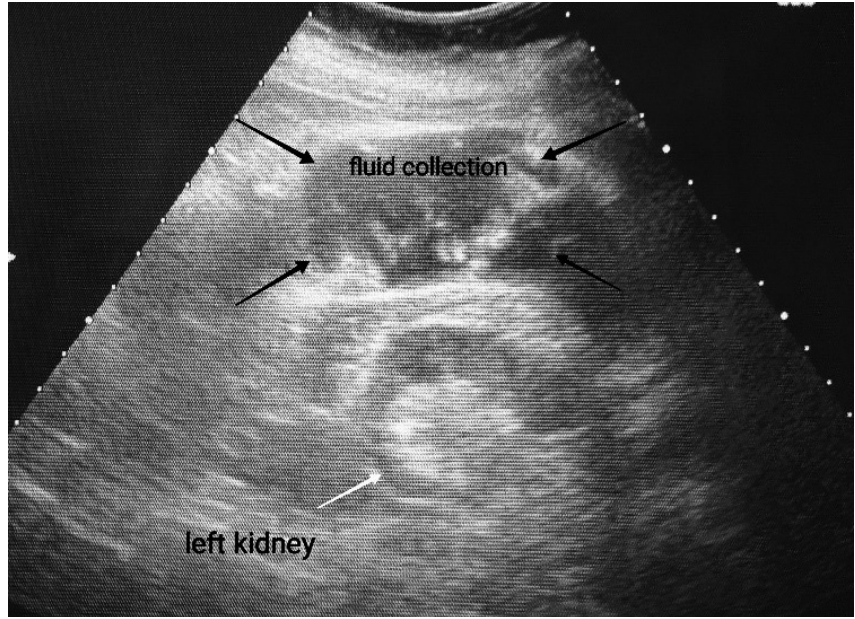

Fig.1. Abdominal sonogram of the patient with acute necrotizing pancreatitis on 7 day after the onset of the disease. Large volume fluid collection behind the descending colon is shown.

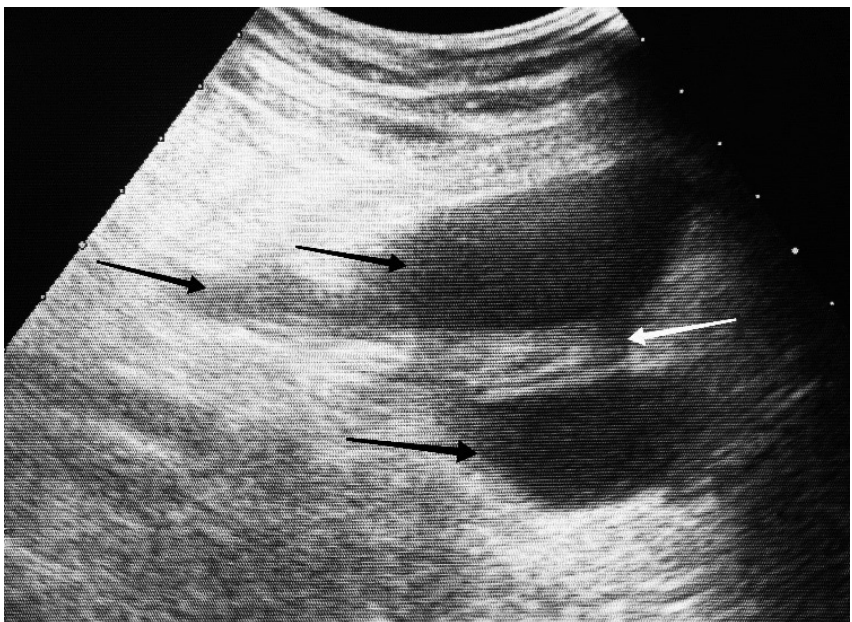

Fig. 3. Abdominal sonogram of patient with a three-chamber fluid collection on the basis of acute necrotic pancreatitis (black arrows indicate the chambers with fluid, white arrow indicates the connecting bridge between them).

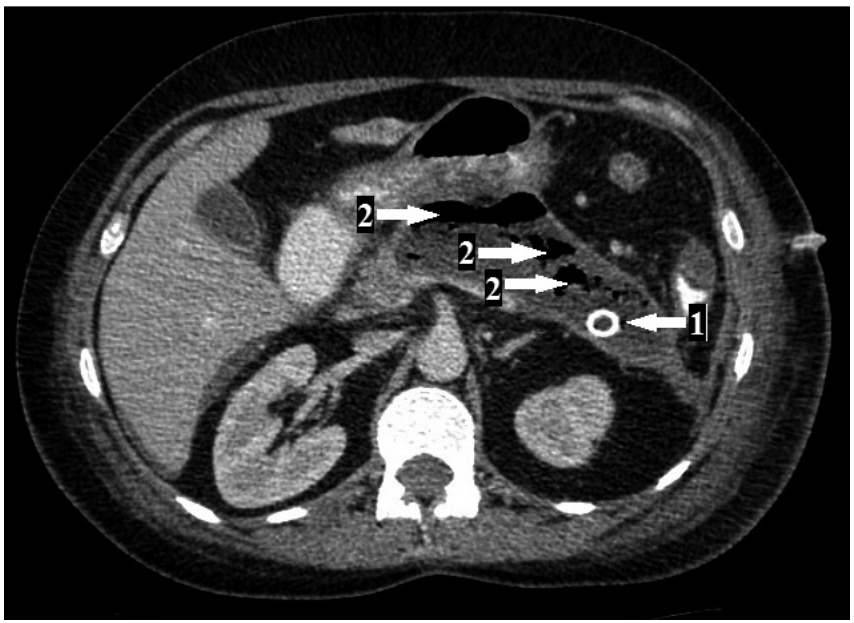

Fig. 5. Tomogram of the abdominal cavity and retroperitoneal space of the patient with acute necrotizing pancreatitis after percutaneous ultrasound-guided puncture and catheter drainage method: 1 - drainage tube in the left side flank. 2 - inhomogeneous structure of the pancreas with the presence of sequestration.

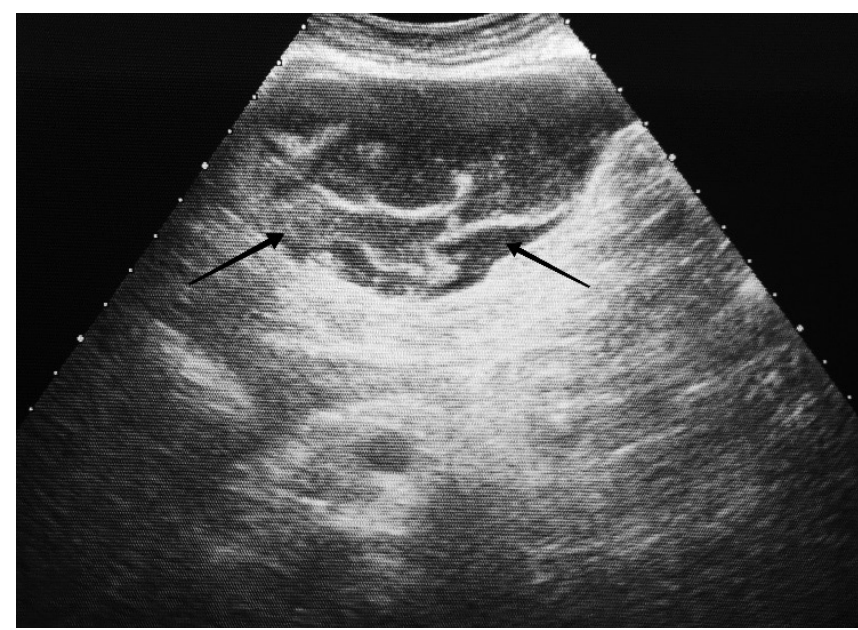

Fig. 2. Abdominal sonogram of the patient with fluid collection with inclusions of detritus due to acute necrotizing pancreatitis, which occurred in the 4th week after the onset of the disease.

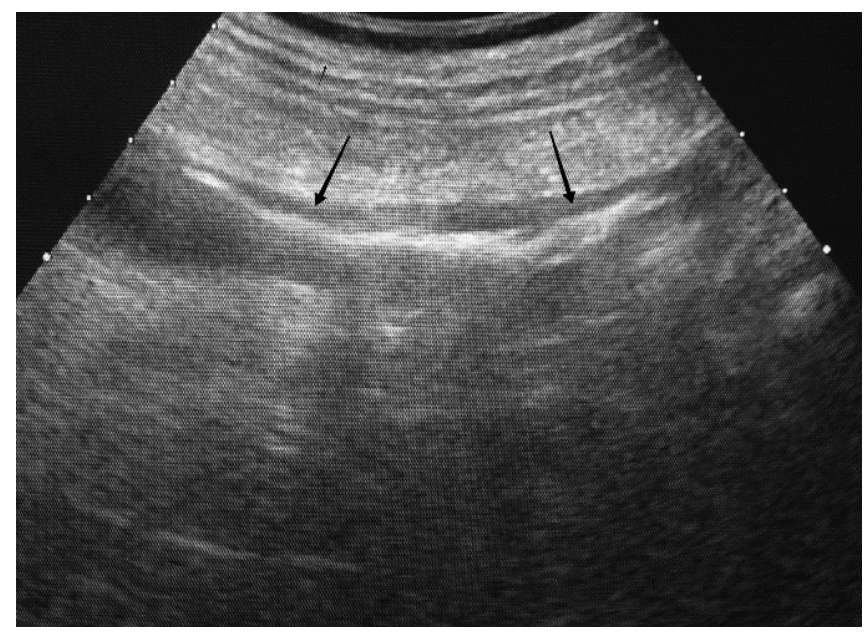

Fig. 4. Abdominal sonogram of the patient immediately after the ultrasound-guided percutaneous catheter drainage method. Drainage is in the central chamber (indicated by black arrows), through which two additional chambers are drained (in the figure, the additional chambers are asleep).

nography was more than $500 \mathrm{ml}$ - puncture of the fluid collection was supplemented by the installation of drainage or paired drainages, depending on the size of the accumulation and fragments of detritus in the liquid (Fig. 2).

In $4(12.5 \%)$ patients a large-size fluid collection (more than $500 \mathrm{ml}$ in volume) had according to ultrasonography heterogeneous inclusions, which were fragments of detritus without signs of intra-abdominal infection. In such patients after percutaneous ultrasound-guided puncture paired drainages with lateral openings were inserted and transdrainage debridement with antiseptic solution was performed. The procedure was performed 3-4 times a day. The efficiency criteria were the change of the nature and the amount of the exudate.

In three $(9.3 \%)$ patients in the comparison group multichamber fluid collections developed. Individual chambers can be connected by "bridges", so it is not always necessary to drain each of the chambers (Fig. 3). It is often enough to adequately drain 
one of them, while the other chambers can drain through the connection. In all three cases, the drainage was installed in the central chamber, the other two were drained through it (Fig. 4).

Signs of infection developed in $2(6.2 \%)$ patients of the comparison group and in $5(14.3 \%)$ patients of the control group $(\mathrm{p}<0.05)$. In case of infection of sterile fluid collections in addition to the classic clinical symptoms (increased pain, hyperthermia, general weakness, violation of the intestinal motility), laboratory parameters (increased leukocytosis with a shift of the formula to the left and signs of endotoxicosis) specific features appeared on sono- and tomograms. The increase in the size of the pancreas, blurring of its contours, loss of homogeneity, the appearance at the site of projection of the gland of inhomogeneous hyperechogenic inclusions, which are sequestral masses - indicated the infection of the process. The appearance of air bubbles was considered an early sign of tissue suppuration (Fig. 5). Suspicion of infection in the absence of ultrasound imaging data were indications for computed tomography.

This study showed that mortality rates in the comparison group are lower than in the control group ( $p>0.05$ ), so the final result in the comparison group with percutaneous ultrasound-guided puncture and catheter drainage method is better. Also in the comparison group, the shorter stay of patients in the hospital $(\mathrm{p}<0.05)$, pain disappeared earlier $(\mathrm{p}<0.05)$, earlier normalized laboratory parameters $(p<0.05)$. Signs of infection were less common in the control group than in the control group $(\mathrm{p}<0.05)$. Laboratory parameters such as leukocytelevels, serum amylase, C-reactive protein were also significantly faster normalized in the group with percutaneous ultrasound-guided puncture and catheter drainage method $(\mathrm{p}<0.05)$, which, in our opinion, was associated with the evacuation of inflammatory fluid rich in enzymes and cytokines.

\section{CONCLUSIONS}

1. Fluid collections around the pancreas belong to the local complications of acute pancreatitis and are recommended for correction by conservative or minimally invasive methods. Indications for percutaneous ultrasound-guided puncture and catheter drainage method are clinical (intraabdominal hypertension and signs of compression of neighboring organs, severe pain, temperature reaction, etc.) and ultrasonographic signs. The presence of fluid on the sonogram more than $500 \mathrm{ml}$, or its rapid appearance in the dynamics are indications for puncture. The presence of a large volume of fluid with heterogeneous inclusions was considered an indication for puncture with following drainage. In the case of heterogeneous fluid content with detrital inclusions through drainage debridement is shown.

2. Percutaneous ultrasound-guided puncture and catheter drainage methods of fluid collections treatment can be used as a step-up approach or independently. The use of ultrasound-guided puncture and catheter drainage methods allows to normalize laboratory (leukocyte, serum amylase, C-reactive protein) and clinical parameters $(\mathrm{p}<0.05)$, reduce infection rates $(\mathrm{p}<0.05)$ and reduce mortality ( $(\mathrm{p}<0.05)$, allows to delay the open surgery with all its risks to the so-called «cold period», which begins after 4 weeks from the onset of the disease. The method requires further study with the possible development of clear algorithms according to the timing after the onset of the disease, the nature of the exudate, the diameter of the drainage tubes, methods of debridement, etc.

\section{REFERENCES}

1. Banks P.A., Bollen T.L., Dervenis C. et al Classification of acute pancreatitis -2012: revision of the Atlanta classification and definitions by international consensus. Gut. 2013; 62: 102-111. doi: 10.1136/ gutjnl-2012-302779.

2. Freeman M., Werner J., van Santvoort H. et al. Interventions for necrotizing pancreatitis summary of a multidisciplinary consensus conference. Pancreas. 2012; 41 (8): 1176-1194; doi: 10.1097/ MPA.0b013e318269c660.

3. Michael G., Christopher M., Susanna D. et al. Minimal access retroperitoneal pancreatic necrosectomy improvement in morbidity and mortality with a less invasive approach. Annals of surgery. 2010; 25 (5): 787-793. doi: 10.1097/SLA.0b013e3181d96c53.

4. Wan J., Wu D., He W. et al. Comparison of percutaneous vs endoscopic drainage in the management of pancreatic fluid collections: A prospective cohort study. Journal of gastroenterology and hepatology. 2020. doi: 10.1111/jgh.15121.

5. Jagielski M., Smoczynski M., Adrych K. et al. Endoscopic necrosectomy under fluoroscopic guidance during transmural drainage of walled-off pancreatic necrosis (WOPN). Polish journal of surgery. 2019; 92 (1): 12-17. doi: 10.5604/01.3001.0013.5610.

6. 10. Huazhi L., Yongzhe W., Chen X. et al. Early ultrasound-guided percutaneous catheter drainage in the treatment of severe acute pancreatitis with acute fluid accumulation. Experimental and therapeutic medicine. 2018; 16: 1753-1757; doi: 10.3892/etm.2018.6398.

7. Leppaniemi A., Tolonen M., Tarasconi A. et al. WSES guidelines for the management of severe acute pancreatitis. World journal of emergency surgery. 2017; 14 (27). doi: 10.1186/s13017-019-0247-0.

8. de Paredes A., Lopez-Duran S., Foruny J. et al. Management of pancreatic collections: an update. Revista espanola de enfermedades digestivas. 2019; 112 (6): 483-490. doi: 10.17235/reed.2020.6814/2019.

9. LiX., Wang L., Tan Z. et al. Percutaneous catheter drainage of pancreatic fluid collections in patients with acute pancreatitis. Indian journal of surgery. 2020. doi: 10.1007/s12262-020-02187-x.

10. Krivoruchko I., Balaka S., Sykal N., Povelichenko M. Treatment of destructive pancreatitis with minimally invasive techniques. Bulletin of VDNZU“Ukrainian Medical Dental Academy». 2013; 13 (1:41):112-114.

11. da Costa D., Boerma D., van Santvoort H. et al. Staged multidisciplinary step-up management for necrotizing pancreatitis. British journal of surgery. 2013; 101 (1): 65-79. doi: 10.1002/bjs.9346.

\section{ORCID and contributionship:}

Tetiana Formanchuk: 0000-0002-9565-8213 A,D

Hryhoriy Lapshyn: 0000-0002-2030-9748 C,D

Oleg Voznyuk: 0000-0002-2518-0429 B,E

Andrii Formanchuk: 0000-0003-4676-1289 B,E

Andrii Zhmur: 0000-0002-8598-5878 ${ }^{F}$

\section{Conflict of interest:}

The Authors declare no conflict of interest. 


\section{CORRESPONDING AUTHOR}

Tetiana Formanchuk

National Pirogov Memorial Medical University

56 Pirogov st., 21018 Vinnytsia, Ukraine

tel: +380674957248

e-mail: mitykt@gmail.com

Received: 11.10 .2020

Accepted: 02.06.2021

A - Work concept and design, B - Data collection and analysis, C - Responsibility for statistical analysis,

$\mathbf{D}$-Writing the article, $\mathbf{E}$-Critical review, $\mathbf{F}$ - Final approval of the article 